\title{
BESSEL POTENTIAL SPACES WITH VARIABLE EXPONENT
}

\author{
Petr Gurka, Petteri HarJulehto And Aleš NeKVindA
}

Abstract. We show that a variable exponent Bessel potential space coincides with the variable exponent Sobolev space if the Hardy-Littlewood maximal operator is bounded on the underlying variable exponent Lebesgue space. Moreover, we study the Hölder type quasi-continuity of Bessel potentials of the first order.

Mathematics subject classification (2000): 46E35, 46E30, $26 \mathrm{D} 10$.

Keywords and phrases: Bessel potential space, Lebesgue space with variable exponent, quasi-continuity.

\section{REFERENCES}

[1] A. Almeida, S. SAmKo, Characterization of Riesz and Bessel potentials of variable Lebesgue spaces, J. Funct. Spaces Appl., 4, (2) (2006), 113-144.

[2] D. Cruz-Uribe, A. Fiorenza And C. J. Neugebauer, The maximal operator on variable $L^{p}$ spaces, Ann. Acad. Sci. Fenn., 28, (2003), 223-238.

[3] D. Cruz-Uribe, A. Fiorenza And C. J. Neugebauer, Corrections to "The maximal operator on variable $L^{p}$ spaces", Ann. Acad. Sci. Fenn., 29, (2004), 247-249.

[4] D. Cruz-Uribe, A. Fiorenza, J. M. MARTEll AND C. PÉREZ, The boundedness of classical operators on variable $L^{p}$ spaces, Ann. Acad. Sci. Fenn., 31, (2006), 239-264.

[5] L. Diening, Maximal function on generalized Lebesgue spaces $L^{p(\cdot)}$, Math. Inequal. Appl., 7, (2) (2004), 245-253.

[6] L. DiEnING, Maximal function on Orlicz-Musiełak spaces and generalized Lebesgue spaces, Bull. Sci. Math., 129, (8) (2005), 657-700.

[7] L. DIENING, Riesz potential and Sobolev embeddings on generalized Lebesgue and Sobolev spaces $L^{p(\cdot)}$ and $W^{k, p(\cdot)}$, Math. Nachr., 263, (1) (2004), 31-43.

[8] L. DiENING, M. RŮŽIČKA, Calderón-Zygmund operators on generalized Lebesgue spaces $L^{p(\cdot)}$ and problems related to fluid dynamics, J. Reine Angew. Math., 563, (2003), 197-220.

[9] D. E. EdmundS, J. RÁKOSNÍK, it Sobolev embeddings with variable exponent, Studia Math., 143, (3) (2000), 267-293.

[10] L. C. Evans, R. F. GARIEPY, Measure Theory and Fine Properties of Functions, CRC Press, Boca Raton, 1992.

[11] P. HARJUlehto, P. Hästö, Lebesgue points in variable exponent spaces, Ann. Acad. Sci. Fenn. Math., 29, (2004), 295-306.

[12] P. Harjulehto, P. Hästö, M. Koskenoja and S. Varonen, it Sobolev capacity on the space $W^{1, p(\cdot)}\left(\mathbb{R}^{n}\right)$, J. Funct. Spaces Appl., 1, (1) (2003), 17-33.

[13] J. Kinnunen, O. Martio, The Sobolev capacity on metric spaces, Ann. Acad. Sci. Fenn. Math., 21, (1996), 367-382.

[14] O. KovÁčIK, J. RÁKosníK, On spaces $L^{p(x)}$ and $W^{1, p(x)}$, Czechoslovak Math. J., 41, (116) (1991), 592-618.

[15] A. K. LERNER, Some remarks on the Hardy-Littlewood maximal function on variable $L^{p}$ spaces, Math. Z., 251, (3) (2005), 509-521.

[16] J. MaLÝ, Hölder type quasicontinuity, Potential Anal., 2, (3) (1993), 249-254. 
[17] A. NeKVINDA, A note on maximal operator on $\ell^{\left\{p_{n}\right\}}$ and $L^{p(x)}(\mathbb{R})$, J. Funct. Spaces Appl., 5, (1) (2007), 49-88.

[18] A. NeKVINDA, Hardy-Littlewood maximal operator on $L^{p(x)}\left(\mathbb{R}^{n}\right)$, Math. Inequal. Appl., 7, (2) (2004), 255-265.

[19] E. NIEMINEN, Hausdorff measures, capacities, and Sobolev spaces with weights, Ann. Acad. Sci. Fenn. Ser. A I Math. Dissertationes, 81, (1991), 39 pp.

[20] L. PICK, M. RŮŽǏ̌KA, An example of a space $L^{p(x)}$ on which the Hardy-Littlewood maximal operator is not bounded, Expo. Math., 19, (2001), 369-371.

[21] M. RŮŽIČKA, Electrorheological Fluids: Modeling and Mathematical Theory, Springer-Verlag, Berlin, 2000.

[22] S. SAMKo, Denseness of $C_{0}^{\infty}\left(\mathbb{R}^{n}\right)$ in the generalized Sobolev spaces $W^{m, p(x)}\left(\mathbb{R}^{n}\right)$, pp. 333-342 in Direct and inverse problems of mathematical physics (Newark, DE, 1997), Int. Soc. Anal. Appl. Comput., bf 5, Kluwer Acad. Publ., Dordrecht, 2000.

[23] E. SteIn, Singular integrals and differentiability properties of functions, Princeton University Press, Princeton, 1970.

[24] H. TRIEBEL, Theory of function spaces, Basel, Birkhäuser, 1983. 